\title{
IDENTIFICATION OF DIFFERENTIALLY EXPRESSED GENES OF SORGHUM [SORGHUM BICOLOR (L.) MOENCH] SEEDLINGS UNDER NITROGEN STRESS BY RNA-SEQ
}

\author{
YANG, G. D. $.^{1,3}-$ ZHOU, Y. F. ${ }^{1 *}-$ HUANG, R. D. ${ }^{1}-$ LIN, F. ${ }^{2}-$ HU, Z. Y. ${ }^{3}-$ HAO, Z. Y. ${ }^{3}-$ \\ LIANG, C. B. ${ }^{4}-$ WANG, Q. ${ }^{5}-$ MENG, X. X. ${ }^{5}-$ DONG, L. D. ${ }^{6}$ \\ ${ }^{1}$ College of Agronomy, Shenyang Agricultural University \\ No.120 Dongling Road, Shenyang 110866 Liaoning, China \\ ${ }^{2}$ College of Bioscience and Biotechnology, Shenyang Agricultural University \\ No.120 Dongling Road, Shenyang 110866 Liaoning, China \\ ${ }^{3}$ Keshan Branch of Heilongjiang Academy of Agricultural Sciences \\ 161005 Heilongjiang, China \\ ${ }^{4}$ Industrial Crops Institute of Heilongjiang Academy of Agricultural Sciences \\ 150086 Heilongjiang, China \\ ${ }^{5}$ Crop Resources Institute of Heilongjiang Academy of Agricultural Sciences \\ 150086 Heilongjiang, China \\ ${ }^{6}$ School of Life Sciences, Guangzhou University, 510006 Guangzhou, China \\ *Corresponding author \\ e-mail:zhouyufei2002@aliyun.com; zhouyufei@syau.edu.cn \\ (Received 22 $2^{\text {nd }}$ Apr 2019; accepted $16^{\text {th }}$ Jul 2019)
}

\begin{abstract}
Sorghum [Sorghum bicolor (L.) Moench] is an important cereal crop with high nitrogen utilization efficiency. In order to analyze the mechanisms of different sorghum genotypes to low-N tolerance and provide the theoretical basis for the breeding of low nitrogen tolerant sorghum varieties, two materials (KZ15 and SZ7) with different tolerance to low-N were treated with nitrogen by hydroponics, the phenotypes were observed, physiological indexes were measured and transcripts were analyzed in this study. Compared to SZ7, KZ15 showed significantly higher capacity of low-N tolerance. The results showed that the change of KZ15 chlorophyll content was lower than that of SZ7 under low-N stress. The activity of nitrate reductase (NR) and glutamine synthetase (GS) had a smaller reduction in KZ15 than SZ7 under low-N stress. High-throughput sequencing revealed several common differentially expressed genes (DEGs) between KZ15 and SZ7, including nitrogen transport and nitrate assimilation genes. In the tolerant genotype KZ15, there were more transcripts related to high affinity nitrate transporters (NRT2.4, NRT3.1 and NRT4.5), suggesting an improved uptake efficiency of inorganic and organic forms of nitrogen. In addition, an abundance of certain transcription factors in the tolerant genotype KZ15 suggests that they may play roles in regulating the response to $\mathrm{N}$-stress. This study will enable us to discover candidate genes potentially useful in improving the resistance to low nitrogen stress by genetic engineering technology and provide theoretical basis for the breeding of low nitrogen tolerant sorghum varieties.
\end{abstract}

Keywords: sorghum, $N$ tolerance, chlorophyll, transcriptome, differentially expressed genes, candidate genes

\section{Introduction}

Nitrogen is a necessary major element for the growth and development of higher plants, which takes part in a series of physiological and biochemical reactions, 
involving various metabolic pathways (Kraiser et al., 2011). There are two mechanisms for obtaining nitrogen in plants: one is to absorb nitrogen nutrition by nodular nitrogenase, which is mainly in leguminous plants. The other is to absorb $\mathrm{NH}^{+}, \mathrm{NO}^{-}$, and other mineral ions from the soil by root transporters. Sorghum mainly absorbs nitrogen through the second mechanism. Studies of nitrogen stress by quantitative trait loci analysis have mainly focused on maize (Gallais et al., 2004), rice (Obara et al., 2004), Arabidopsis (Loudet et al., 2003a,b) and sorghum (Gelli et al., 2017). At present, the study in low nitrogen tolerance of sorghum has lagged behind other grain crops (Rosa et al., 2014). Few studies of the answers of the molecular response mechanisms of growth and development to nitrogen deficiency of sorghum have been reported.

In this study, the phenotypic and transcriptomic responses of two sorghum varieties, KZ15 and SZ7, under low nitrogen stress and control were investigated. Unraveling mechanisms underlying low nitrogen tolerance of sorghum may provide useful information to genetic engineering and improve the low nitrogen tolerance of sorghum. Such knowledge can also be useful for increasing nitrogen use efficiency for sustainable agriculture.

\section{Materials and methods}

\section{Plant materials and growth conditions}

Two sorghum varieties, Keza15 (KZ15, tolerant to nitrogen starvation) and Suiza7 (SZ7, sensitive to nitrogen starvation), were used for this study, which were provided by the Keshan branch of the Heilongjiang Academy of Agricultural Sciences.

This experiment was conducted in the greenhouse of Keshan Branch of Heilongjiang Academy of Agricultural Sciences in China. Uniform and plump-eared sorghum seeds were selected and germinated under dark conditions after disinfection, and then sown in vermiculite.

\section{Stress treatments and sample preparations}

Trifoliate seedlings were moved into foam boxes wrapped in black plastic film and then cultured with Hoagland nutrient solution. The average temperature and light-dark period were $25^{\circ} \mathrm{C} / 20^{\circ} \mathrm{C}$ and $14 \mathrm{~h} / 10 \mathrm{~h}$. $\mathrm{CaCl}_{2}$ was added to supplement $\mathrm{Ca}^{2+}$. Control plants (in the solution of $4 \mathrm{mmol} / \mathrm{L} \mathrm{Ca}\left(\mathrm{NO}_{3}\right)_{2}$ ) were grown in parallel and collected at the same time points. The $\mathrm{pH}$ value was adjusted to 5.8 (Ma, 2014). KA, SA, KB and SB indicated the seedlings of KZ15 and SZ7 in low nitrogen treatment and control, respectively. The process was repeated in triplicate. Leaves of seedling which treated for seven days were taken as samples.

The method of the determination of chlorophyll content was referred to the modified method cited by Wang et al. (2006). The following formulas were used to calculate the content of photosynthetic pigments, in which $\mathrm{V}$ and $\mathrm{W}$ indicated the volume of extracting liquid (ml) and the weight of material $(\mathrm{g})$.

$$
\begin{aligned}
& \text { Chlorophyll a }(\mathrm{Ca}) / \mathrm{mg} \cdot \mathrm{g}-1=(12.21 \mathrm{~A} 663-2.81 \mathrm{~A} 646) \mathrm{V} /(1000 \times \mathrm{W}) \\
& \text { Chlorophyll b }(\mathrm{Cb}) / \mathrm{mg} \cdot \mathrm{g}-1=(20.13 \mathrm{~A} 646-5.03 \mathrm{~A} 663) \mathrm{V} /(1000 \times \mathrm{W})
\end{aligned}
$$


The method of nitrate reductase determination was referred to Rajasekhar et al. (1986). The determination of glutamine synthase (GS) was referred to the method of Ding et al. (2006).

The experiment was performed using three biological replicates, and differences were statistically analyzed using Student's t-test $\left({ }^{*} \mathrm{P}<0.05,{ }^{* *} \mathrm{P}<0.01\right)$. Bars indicate standard error of the mean. Microsoft Excel 2010 and SPSS 18.0 software are employed for data collation and analysis.

\section{RNA seq analysis}

Total RNA was isolated from each sample. Quality and quantity evaluation of total RNA, library constructing, and Illumina sequencing were performed in the Beijing Genome Institute (BGI, Shenzhen, China).

\section{Transcript data analysis}

TopHat 2 (Kim et al., 2015) was used to compare transcripts with reference genomes. After genome comparison, the StringTie tool was used to identify exonic regions, and then the transcript was reconstructed. The transcript was reconstructed by the DeNovo assembly method. The actual transcripts were screened out, and the low expression transcripts were filtered by the TPM value (filtering criteria: TPM $>0.5$ in at least one sample).

Differential expressed genes between samples were defined by DESeq using two separate models (Anders and Huber, 2010), based on fold change $>2$ and false discovery rate-adjusted $\mathrm{P}$ value $<0.05$.

According to the result of GO annotation, DEGs were classified to GO terms according to official classification. Phyper was used to perform GO functional enrichment. The p-value calculating formula in hyper geometric test was:

$$
P=1-\sum_{i=0}^{m-1} \frac{\left(\begin{array}{l}
M \\
i
\end{array}\right)\left(\begin{array}{l}
N-M \\
n-i
\end{array}\right)}{\left(\begin{array}{l}
N \\
n
\end{array}\right)}
$$

Then, false discovery rate (FDR) was calculated for each p-value. In general, the term FDR $<=0.01$ was defined as significantly enriched.

\section{Results}

\section{Phenotypic investigation of KZ15 and SZ7 under low-N stress}

To evaluate the impact of low-N stress on sorghum, seedlings were planted in an intelligent greenhouse with low-N stress $(0 \mathrm{mmol} / \mathrm{L})$ and under control conditions ( $4 \mathrm{mmol} / \mathrm{L}$ ). Under low-N stress, the seedlings of SZ7 leaves turned pale yellow, but the seedlings of KZ15 leaves did not significantly change (Fig. 1A and 1B). Subsequently, chlorophyll content, activity of nitrate reductase (NR) and glutamine synthetase (GS) were measured in both KZ15 and SZ7 of the low-N treatment and control. The results showed that the seedlings of SZ7 have a $28.77 \%$ average decrease in total chlorophyll content, but the seedlings of KZ15 only have $12.39 \%$ average decrease (Fig. IC). 
Compared to the control, the content of chlorophyll a and chlorophyll b in SZ7 showed a $27.32 \%$ and $33.66 \%$ decrease, respectively. But the content of that in KZ15 was $8.86 \%$ and $22.71 \%$, respectively (Fig. $1 D, 1 E$ ). These results demonstrate that the change of KZ15 chlorophyll content was lower than that of SZ7 under low-N stress, and chlorophyll $\mathrm{b}$ content was more sensitive under low-N stress than the content of chlorophyll a. In addition, the activity of NR and GS was significantly reduced in SZ7 under low-N stress, but the activity of NR and GS exhibited little change in KZ15 under low-N stress (Fig. $1 F, 1 G$ ). These results demonstrate that KZ15 is more N-stress tolerant compared with SZ7.

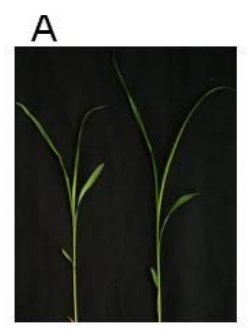

$\mathrm{Ka} \quad \mathrm{Kb}$

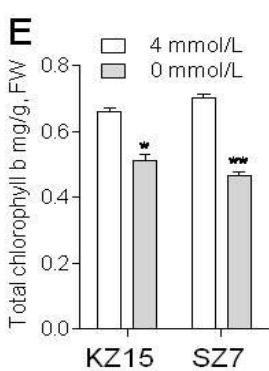

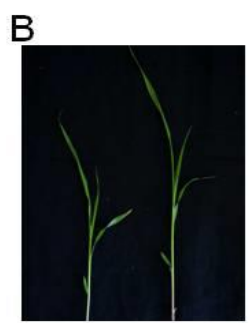

$\mathrm{Sa} \quad \mathrm{Sb}$
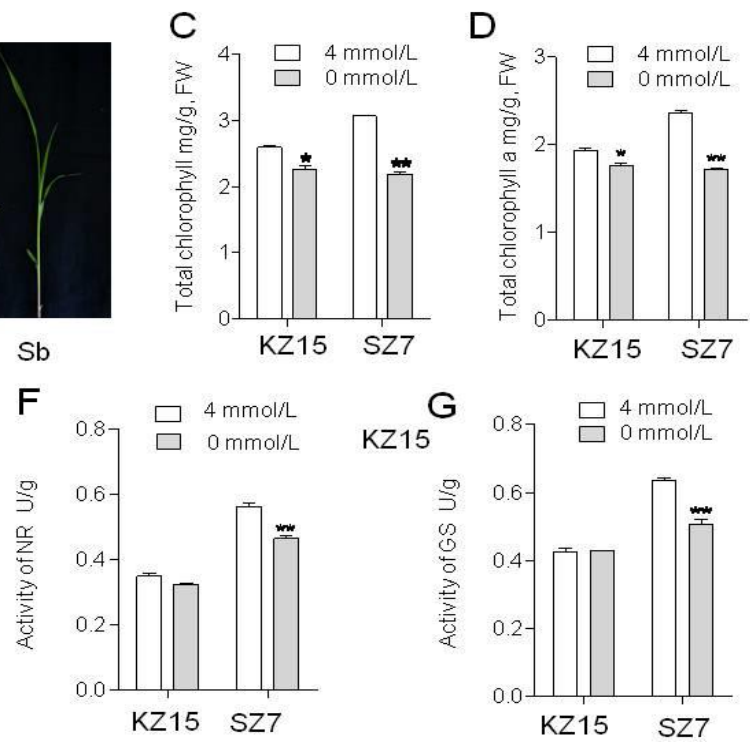

Figure 1. Morphological performance of KZ15 and SZ7 measured under low-N treatment and non-stress concrol nitrogen concentration treatment. $(A, B)$ Phenotype of KZ15 and SZ7 under low-N treatment. (C) Total chlorophyll content. (D) Chlorophyll a content. (E) Chlorophyll b content. ( $F)$ Activity of NR. (G) Activity of GS. ( $* P<0.05 ; * * P<0.01)$. The experiment was performed using three biological replicates, and differences were statistically analyzed using

Student's $t$-test $\left(* P<0.05\right.$, $\left.{ }^{*} P<0.01\right)$. Bars indicate standard error of the mean

\section{Summary of transcriptomic profiling sequencing}

Both full-N and low-N treatments were used to analyze the response of sorghum seedlings to nitrogen stress. Twelve libraries were constructed and sequenced. After removing low-quality reads, an average of $24.09 \mathrm{Mb}$ (range of 24.04 to $24.11 \mathrm{Mb}$ ) clean reads were obtained in each library, in which more than $86.27 \%$ of clean reads per library could be mapped to the reference sorghum genome (Table 1). The Q30 values were higher than $90 \%$, which indicated that the sequencing quality was good. The GC content of 12 samples ranged from 52.11\% to 55.24\%. This result indicated that the sequencing data could be used for further analysis.

\section{Special and common low-N-responsive DEGs between KZ15 and SZ7}

To examine the special and common DEGs between KZ15 and SZ7 under low-N treatment, a Venn diagram were constructed. Many common DEGs were identified in KZ15 and SZ7. In total, 793 DEGs were common low-N-responsive genes (Fig. 2A), 
among which 277 were upregulated (Fig. 2B) and 486 were downregulated (Fig. 2C). Under low-N treatment, 404 DEGs were found specifically upregulated in KZ15 and 762 DEGs were specifically upregulated in SZ7. In addition, 642 and 1132 DEGs were specifically downregulated in KZ15 and SZ7, respectively (Fig. 2A, 2B).

Table 1. Categorization and abundance of transcripts detected with RNA-seq

\begin{tabular}{c|c|c|c|c|c|c}
\hline Sample & $\begin{array}{c}\text { Total Raw } \\
\text { Reads }(\mathbf{M b})\end{array}$ & $\begin{array}{c}\text { Total Clean } \\
\text { Reads }(\mathbf{M b})\end{array}$ & $\begin{array}{c}\text { Clean Reads } \\
\text { Q30 }(\boldsymbol{\%})\end{array}$ & $\begin{array}{c}\text { Mapping Ratio to } \\
\text { genome }\end{array}$ & $\begin{array}{c}\text { Mapping Ratio } \\
\text { to genes }\end{array}$ & GC $(\boldsymbol{\%})$ \\
\hline ka_1 & 24.14 & 24.11 & 90.34 & $94.58 \%$ & $86.57 \%$ & 53.21 \\
ka_2 & 24.14 & 24.10 & 90.95 & $94.64 \%$ & $86.58 \%$ & 53.47 \\
ka_3 & 24.14 & 24.11 & 90.88 & $94.37 \%$ & $86.27 \%$ & 52.11 \\
kb_1 & 24.14 & 24.07 & 91.42 & $94.70 \%$ & $86.99 \%$ & 54.23 \\
kb_2 & 24.14 & 24.11 & 91.28 & $94.83 \%$ & $87.26 \%$ & 53.85 \\
kb_3 & 24.14 & 24.11 & 91.52 & $94.88 \%$ & $87.35 \%$ & 53.75 \\
sa_1 & 24.14 & 24.11 & 91.08 & $94.96 \%$ & $86.91 \%$ & 53.11 \\
sa_2 & 24.14 & 24.11 & 90.73 & $95.20 \%$ & $87.28 \%$ & 52.95 \\
sa_3 & 24.14 & 24.04 & 90.71 & $94.96 \%$ & $86.91 \%$ & 55.24 \\
sb_1 & 24.14 & 24.07 & 91 & $94.83 \%$ & $86.75 \%$ & 54.73 \\
sb_2 & 24.14 & 24.08 & 90.79 & $95.20 \%$ & $87.14 \%$ & 55.31 \\
sb_3 & 24.14 & 24.08 & 90.7 & $94.75 \%$ & $86.51 \%$ & 54.53 \\
\hline
\end{tabular}

A
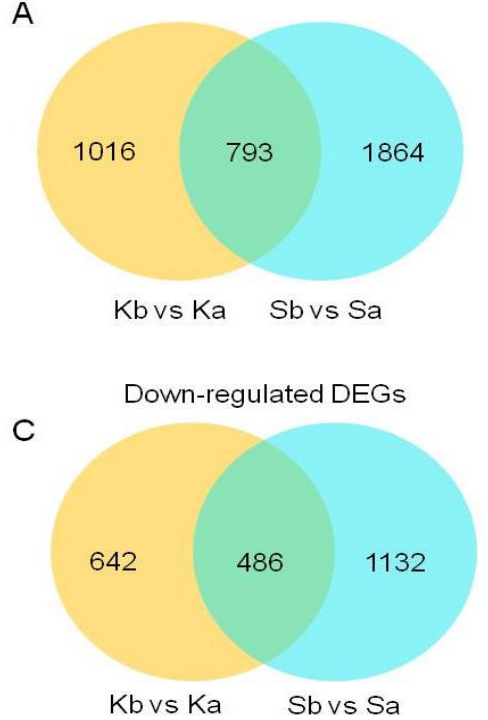

B Up-regulated DEGs

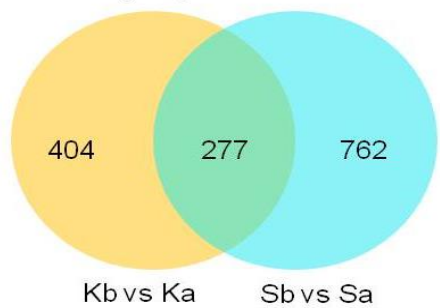

Kbvs Ka Sbvs Sa

Figure 2. Venn diagrams of differentially expressed genes (DEGs) after nitrogen deficiency., (A).Venn diagram showing the common DEGs between KZ15 and SZ7., (B).Venn diagram showing the special and common up-regulated DEGs between KZ15 and SZ7., (C).Venn diagram showing the special and common down-regulated DEGs between KZ15 and SZ7

\section{GO enrichment of DEGs}

The DEGs were categorized into 46 functional groups in KZ15 and SZ7. According to the significance and reliability, GO entries with significant enrichment were screened and simplified. Twenty-six GO entries with the highest enrichment significance were screened out in KZ15 (Table 2), and 11 GO items with the highest significance were selected in SZ7 (Table 3). In biological process, GO terms related to metabolic process were the most enriched both in tolerant cultivar KZ15 (513) and sensitive cultivar KZ15 


$$
-11530 \text { - }
$$

(731). DEG transcripts associated with stress responses included drug decomposition process in sensitive genotypes. DEGs associated with the metabolic process of pigmentation and glutathione were enriched in tolerant genotypes.

Table 2. GO analysis of genes specifically expressed in KZ15 after $N$-starvation

\begin{tabular}{c|c|c|c|c}
\hline GO term & Ontology & Description & Gene Number & P-value \\
\hline GO:0009522 & C & Photosynthetic system I & 10 of 720 & 0.00022 \\
GO:0005576 & C & Extracellular domain & 64 of 720 & 0.00025 \\
GO:0009521 & C & Photosynthetic system & 11 of 720 & 0.0439 \\
GO:0016491 & F & Oxidoreductase activity & 179 of 944 & $1.97 \mathrm{E}-11$ \\
GO:0003824 & F & Catalytic activity & 663 of 944 & $6.69 \mathrm{E}-06$ \\
GO:0048037 & F & Cofactor binding & 114 of 944 & $9.42 \mathrm{E}-06$ \\
GO:0046527 & F & Glucose transferase activity & 38 of 944 & $2.51 \mathrm{E}-05$ \\
GO:0080043 & $\mathrm{F}$ & 3- Quercetin Glucosyl transferase activity & 27 of 944 & $2.92 \mathrm{E}-05$ \\
GO:0080044 & $\mathrm{F}$ & 7- Quercetin Glucosyltransferase activity & 27 of 944 & $2.92 \mathrm{E}-05$ \\
GO:0046906 & $\mathrm{F}$ & Tetrapyrrol binding & 62 of 944 & 0.00015 \\
GO:0035251 & $\mathrm{F}$ & UDP- Glucose transferase activity & 31 of 944 & 0.00049 \\
GO:0016168 & $\mathrm{F}$ & Chlorophyll binding & 8 of 944 & 0.00787 \\
GO:0020037 & $\mathrm{F}$ & Heme binding & 54 of 944 & 0.01346 \\
GO:0004364 & $\mathrm{F}$ & Glutathione transferase activity & 17 of 944 & 0.02068 \\
GO:0042440 & $\mathrm{P}$ & Pigment Metabolism & 18 of 715 & $6.47 \mathrm{E}-05$ \\
GO:0019748 & $\mathrm{P}$ & Secondary metabolic process & 40 of 715 & 0.00027 \\
GO:0015994 & $\mathrm{P}$ & Metabolism of chlorophyll & 11 of 715 & 0.00087 \\
GO:0006778 & $\mathrm{P}$ & Metabolism of Porphyrin-containing & 14 of 715 & 0.00251 \\
GO:0051186 & $\mathrm{P}$ & Compounds & 60 of 715 & 0.00625 \\
GO:0006749 & $\mathrm{P}$ & Cofactor metabolic process & 18 of 715 & 0.01025 \\
GO:0033013 & $\mathrm{P}$ & Glutathione Metabolism & 14 of 715 & 0.01182 \\
GO:0009765 & $\mathrm{P}$ & Tetrapyrrol Metabolism & 9 of 715 & 0.01922 \\
GO:0018298 & $\mathrm{P}$ & Photosynthesis, Light Harvest & 8 of 715 & 0.02056 \\
GO:0009407 & $\mathrm{P}$ & Protein chromophore linkage & 11 of 715 & 0.02232 \\
GO:0090487 & $\mathrm{P}$ & Toxin decomposition process & 11 of 715 & 0.02232 \\
GO:0046148 & $\mathrm{P}$ & Secondary metabolite decomposition process & 12 of 715 & 0.02666 \\
\hline
\end{tabular}

C: Cellular component; P: Biological process; F: Molecular function

Table 3. GO analysis of genes specifically expressed in SZ7 after $N$-starvation

\begin{tabular}{c|c|c|c|c}
\hline GO term & Ontology & Description & Gene Number & p-value \\
\hline GO:0005576 & C & Extracellular domain & 87 of 1111 & 0.00114 \\
GO:0009522 & C & Photosynthetic system I & 11 of 1111 & 0.00263 \\
GO:0009521 & C & Photosynthetic system & 16 of 1111 & 0.00485 \\
GO:0016491 & F & Oxidoreductase activity & 239 of 1407 & $3.12 \mathrm{E}-10$ \\
GO:0046906 & F & Tetrapyrrol binding & 90 of 1407 & $8.89 \mathrm{E}-07$ \\
GO:0048037 & F & Cofactor binding & 156 of 1407 & $9.07 \mathrm{E}-06$ \\
GO:0020037 & F & Heme binding & 83 of 1407 & $2.19 \mathrm{E}-05$ \\
GO:0003824 & F & catalytic activity & 951 of 1407 & 0.00074 \\
GO:0004497 & F & Monooxygenase activity & 55 of 1407 & 0.02333 \\
GO:0042737 & P & Drug decomposition process & 47 of 1019 & 0.00837 \\
GO:0051187 & P & Cofactor catabolism & 35 of 1019 & 0.01595 \\
\hline
\end{tabular}

C: Cellular component; P: Biological process; F: Molecular function

In molecular function ontology, catalytic activity was the term which was enriched and most abundant in both KZ15 (633) and SZ7 (951). The terms oxidoreductase activity, cofactor binding, tetrapyrrole binding and heme binding were found in both KZ15 and SZ7. GO terms belonging to molecular functions such as glutathione transferase activity, chlorophyll binding, glucose transferase activity, 3-quercetin 
glucosyl transferase activity, and UDP-glucose transferase activity were enriched in KZ15. With respect to cellular component ontology, 64 genes were enriched in the extracellular region, followed by the photosynthetic pathway, suggesting that genes differentially expressed in the photosynthetic system and extracellular region of KZ15 may play an important role in low nitrogen tolerance. In SZ7, the most significant Go term was extracellular domain (87 genes). The other two GO terms (Photosynthetic system I and photosynthetic system) were related to the photosynthetic system.

\section{Functional enrichment of significant genes}

In this study, KEGG pathway enrichment analysis of DEGs of KZ15 and SZ7 under nitrogen starvation were carried out. In KZ15, a total of 1526 genes were found to be enriched in 124 pathways, which were divided into five branches: cell process, genetic information processing, environmental information processing, metabolism, and biological system. The results showed that nitrogen starvation had certain effects on the biosynthesis of secondary metabolites, carbohydrates, amino acids, lipids, terpenoids, ketones, cofactors, vitamins, plant signal transduction, membrane transport, protein folding, sorting, degradation, and DNA transcription. In addition, there were 125 DEGs that were related to environmental adaptation. In this study, the top ten pathways with the highest enrichment level were analyzed (Table 4). We found that the difference of nitrogen treatments caused changes of genes related to secondary metabolites biosynthesis, porphyrin metabolism, chlorophyll metabolism, photosynthetic antenna protein pathways, and other pathways, suggesting that these substances may be related to the absorption or metabolism of nitrogen in KZ15.

Table 4. Top 10 KEGG pathway in KZ15 after $N$-starvation

\begin{tabular}{c|c|c|c|c|c}
\hline No. & Pathway ID & Pathway & DEGs genes & Pvalue & Qvalue \\
\hline 1 & ko01110 & $\begin{array}{c}\text { Secondary Metabolites Biosynthesis } \\
\text { Porphyrin and chlorophyll } \\
\text { metabolism }\end{array}$ & 283 & $6.83 \mathrm{E}-15$ & $8.47 \mathrm{E}-13$ \\
2 & ko00860 & 19 & $2.70 \mathrm{E}-07$ & $1.67 \mathrm{E}-05$ \\
3 & ko00196 & Photosynthetic antenna protein & 8 & $9.05 \mathrm{E}-06$ & $3.74 \mathrm{E}-04$ \\
4 & ko01100 & Metabolic pathway & 380 & $1.65 \mathrm{E}-05$ & $4.31 \mathrm{E}-04$ \\
5 & ko00250 & Metabolism of alanine, aspartic acid & 16 & $1.74 \mathrm{E}-05$ & $4.31 \mathrm{E}-04$ \\
6 & ko00220 & and glutamic acid & 15 & $2.15 \mathrm{E}-05$ & $4.44 \mathrm{E}-04$ \\
7 & ko00941 & Arginine biosynthesis & 22 & $6.12 \mathrm{E}-05$ & $1.08 \mathrm{E}-03$ \\
8 & ko04712 & Plavonoid biosynthesis & 31 & 0.000188563 & $2.92 \mathrm{E}-03$ \\
9 & ko00350 & Tyrosine metabolism & 15 & 0.000289618 & $3.67 \mathrm{E}-03$ \\
10 & ko00908 & Zeatin biosynthesis & 10 & 0.000301345 & $3.67 \mathrm{E}-03$ \\
\hline
\end{tabular}

In SZ7, a total of 1694 genes were found to be enriched in 119 pathways. The results showed that nitrogen treatment had certain effects on the metabolism of carbohydrates, secondary metabolites, amino acids, lipids, terpenoids, ketones, cofactors, microorganisms, plant signal transduction, membrane transport, and DNA transcription and translation. A total of 161 DEGs were related to environmental adaptation. In this study, the top ten pathways with the highest enrichment level were analyzed (Table 5). We found that the difference of nitrogen level in SZ7 caused the different expression of genes related to the biosynthesis of secondary metabolites, monoterpenes, carotenoids, content of amino acids, starch, and sucrose, suggesting that these substances might be related to the metabolism of nitrogen uptake of SZ7. 
Table 5. Top 10 KEGG pathway in SZ7 after $N$-starvation

\begin{tabular}{c|c|c|c|c|c}
\hline No. & Pathway ID & Pathway & DEGs genes & Pvalue & Qvalue \\
\hline 1 & ko01110 & Secondary Metabolites Biosynthesis & 344 & $5.12 \mathrm{E}-07$ & $6.46 \mathrm{E}-05$ \\
2 & k000902 & Monoterpene biosynthesis & 9 & $7.72 \mathrm{E}-05$ & $4.86 \mathrm{E}-03$ \\
3 & k000906 & carotenoid biosynthesis & 18 & 0.000176 & $7.40 \mathrm{E}-03$ \\
4 & ko00904 & Diterpenoid biosynthesis & 18 & 0.000853 & $2.15 \mathrm{E}-02$ \\
5 & ko00350 & Tyrosine metabolism & 18 & 0.000853 & $2.15 \mathrm{E}-02$ \\
6 & ko00250 & Metabolism of alanine, aspartic acid & 16 & 0.001541 & $3.24 \mathrm{E}-02$ \\
7 & k000500 & and glutamic acid & 67 & 0.002004 & $3.34 \mathrm{E}-02$ \\
8 & ko01100 & Metabolic pathway & 519 & 0.002124 & $3.34 \mathrm{E}-02$ \\
9 & ko00950 & Biosynthesis of Isoquinoline & 13 & 0.003257 & $4.56 \mathrm{E}-02$ \\
10 & klkaloids & 11 & 0.00804 & $1.01 \mathrm{E}-01$ \\
\hline
\end{tabular}

\section{Genes involved in nitrogen metabolism and glutathione metabolism}

The results of GO enrichment analysis indicated the common low-N responses on nitrogen metabolism and the processes of glutathione metabolism in both KZ15 and SZ7. Nitrogen metabolism was composed of nitrogen transport and nitrate assimilation. In total, 16 genes participated in nitrogen metabolism, including nitrate reductase, ferredoxin-nitrite reductase, ureide permease 2, chloride channel protein and the NRT1/ PTR FAMILY (NRT2.2, NRT2.3, NRT3.1, NRT4.3, NRT4.4, and NRT6.3), which were all upregulated in both KZ15 and SZ7 under N-starvation. Only five genes (IN2-1, NRT4.3, NRT6.3, NRT8.3, and NRT8.5) encoding nitrate transport proteins were downregulated after N-starvation (Figure 3A).
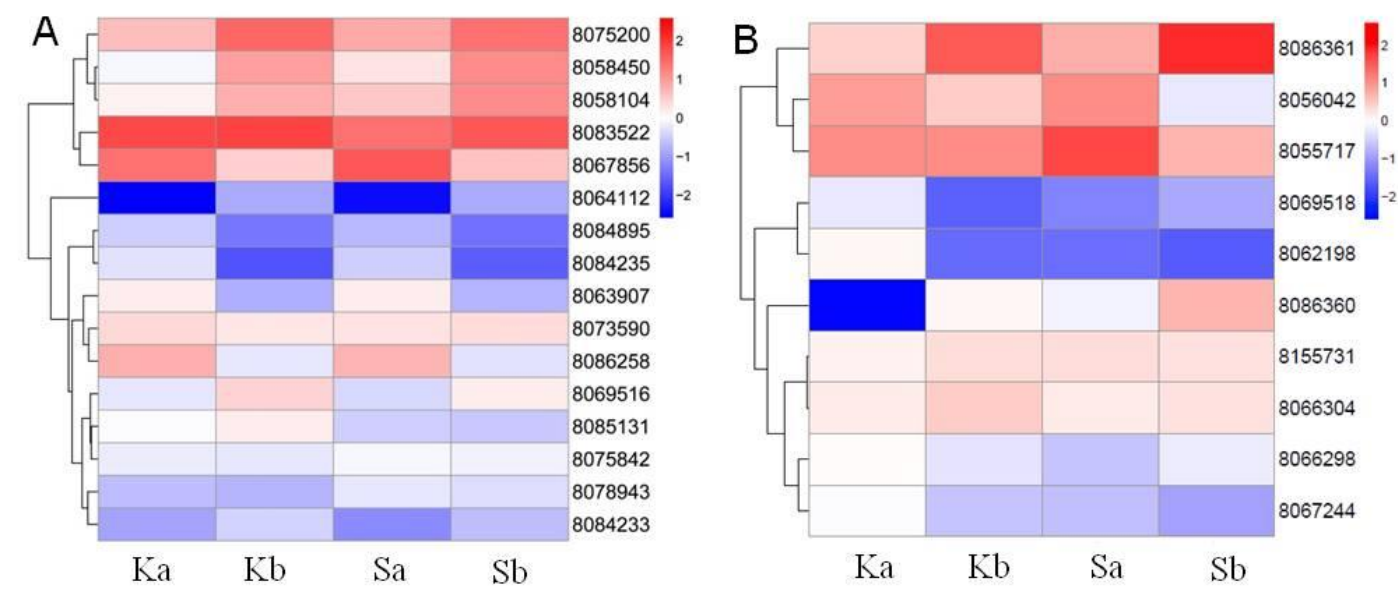

Figure 3. Heatmap of differentially expressed genes (DEGs) related to Nitrogen metabolism (A) and Glutathione metabolic (B) in KZ15 and SZ7 under N-starvation

Although similar expression pattern of genes involved in nitrogen metabolism existed in both varieties under N-starvation, the gene expression pattern in cell redox homeostasis was distinct. Among these, eight DEGs mapped to the cell redox homeostasis pathway and glutathione metabolism was found to be upregulated in KZ15 and SZ7 under N-starvation. Only two DEGs had the opposite expression (Figure 3B). 


\section{DEG transcripts abundant in KZ15 under N-starvation}

Genes that are differentially expressed in only one variety should be important for the phenotypic differences between KZ15 and SZ7 under N-starvation. A total of 389 genes that were especially up-regulated in KZ15 were functionally involved in low$\mathrm{N}$ tolerance. The expression of DEGs related to nitrogen metabolism and defense response was identified, which included genes associated with nitrate uptake and assimilation, transcriptional regulation, hormone-signaling, and oxidative stress. The results of RNA-seq for known nitrogen transport and assimilation genes indicated that $\mathrm{N}$-stress may increase the abundance of genes encoding high affinity nitrate transporters in tolerant cultivars KZ15. For example, genes encoding the nitrate transporters NRT2.4, NRT3.1, and NRT4.5 were more abundant in the tolerant genotype KZ15 than SZ7 (Figure 4A).
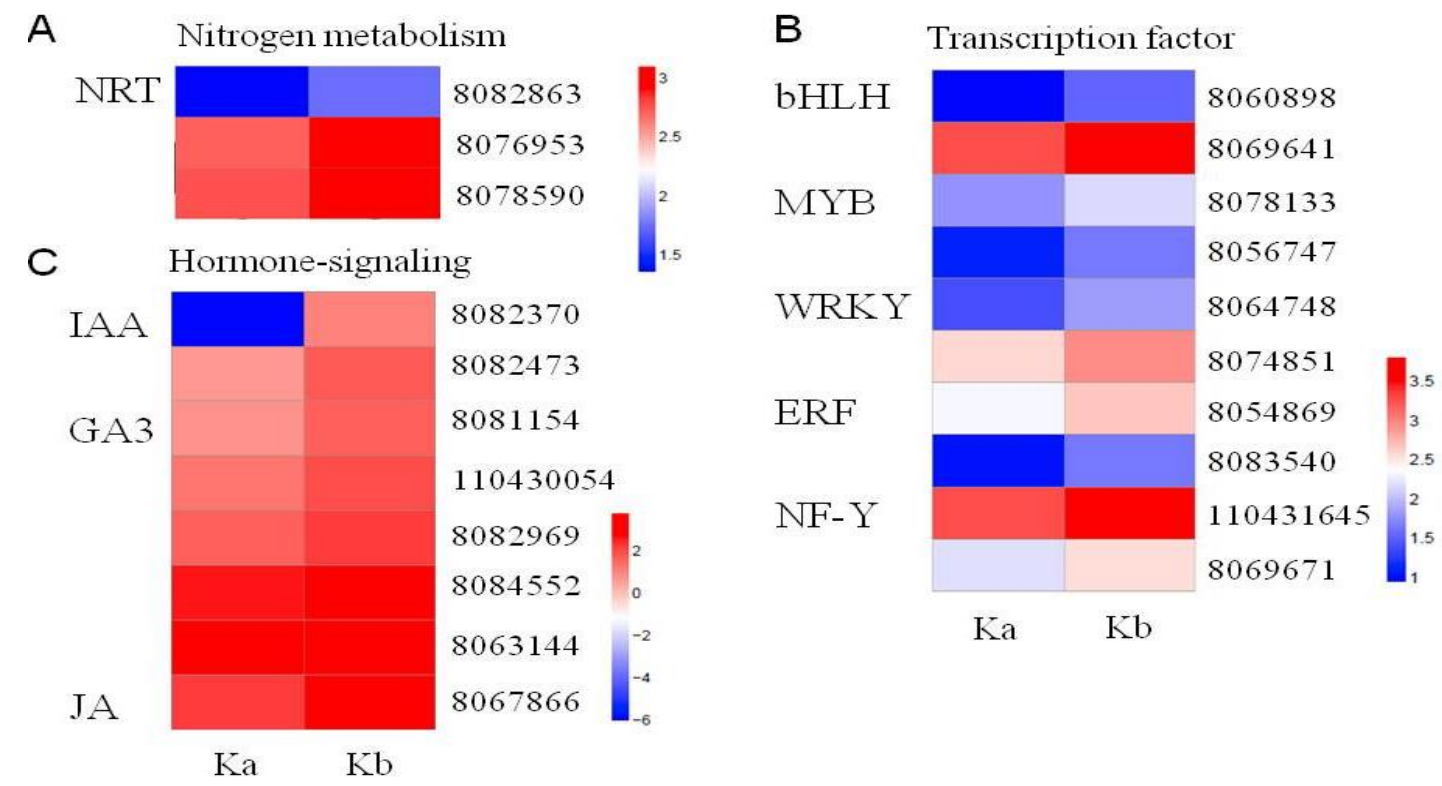

Figure 4. Heatmap of differentially expressed genes( DEGs) related to low-N tolerance responses in KZ15. (A) Nitrogen metabolism. (B) Transcription factors

Transcription factors are important in molecular mechanisms regulating growth, developmental processes, and responses to biotic or abiotic stress. In this study, a total of ten transcription factor (TF) encoding genes were especially upregulated in KZ15 under N-starvation. These transcription factors included the WRKY, MYB(myeloblastosis), bHLH(b helix-loop-helix), ERF (ethylene responsive factor), and NF-Y(nuclear factor-Y) transcription factor families (Figure $4 B$ ). In addition, several genes associated with hormone-signaling pathways were upregulated in the tolerant genotype KZ15 under N-starvation, such as auxin responsive GH3(Gretchen Hagen 3) gene, auxin-responsive protein IAA30, gibberellin receptor GID1(Gibberellin insensitive dwarf 1), gibberellin 2-oxidase, and jasmonate ZIM (Zinc finger gene) domain-containing protein (Figure $4 C$ ). DEGs involved in oxidative stress response and flavonoid metabolites were also upregulated in KZ15 under N-stress. 


\section{Discussion}

\section{Changes of physiology and expressing genes in sorghum under low-N stress}

The results of this study showed that the DEGs in chloroplast of SZ7 were significantly more abundant than that of KZ15. When nitrogen was deficient, physiological analysis also showed that the chlorophyll content of SZ7 was significantly lower than that of the normal nitrogen application, while the chlorophyll content of KZ15 did not change significantly under different nitrogen treatments. The activity of NR and GS decreased under low nitrogen, but the decrease of KZ15 was lower than that of SZ7. The decrease of low nitrogen tolerance sorghum was lower than that of low nitrogen sensitivity sorghum. The results of this study are consistent with previous studies on maize (Presterl et al., 2010), wheat (Han et al., 2007; Xiong et al., 2016), and rice (Zeng et al., 2007).

The results of RNA-sequencing revealed a series of DEGs between KZ15 and SZ7. To understand the molecular mechanisms of these two sorghum cultivars with significantly different nitrogen uptake levels, we found that 681 genes and 1039 genes were upregulated in KZ15 and SZ7 without nitrogen application, respectively, and the number of downregulated genes were 1128 and 1618, respectively. As an $\mathrm{N}^{+}$starvation sensitive genotype, the results showed that the DEGs of SZ7 were significantly more abundant than those of KZ15 in the process of organic nitrogen biosynthesis and nitrogen metabolism, which is similar to previous studies in maize and Arabidopsis thaliana (Gallais and Hirel, 2004). We also found that both cultivars were involved in photosynthesis and protein differential expression. Similar phenomena have been found in the gene expression profile of Arabidopsis thaliana (Himanen et al., 2004).

\section{Special and common Low-N-responsive DEGs in sorghum under low-N stress}

In this study, many DEGs were identified in both sorghum varieties which may be a response to low-N stress. Previous studies have shown that many DEGs associated with high affinity nitrate transporters (NRT2.2, NRT2.3, NRT2.5, and NRT2.6) were identified in low-N tolerant sorghum varieties (Gelli et al., 2014). Consistent with this, we found that the transcript encoding nitrate transporters NRT2.4, NRT3.1, and NRT4.5 were abundant in the tolerant genotype KZ15 compared to SZ7. We also found that the NRT1/ PTR family (NRT2.2, NRT2.3, NRT3.1, NRT4.3, NRT4.4, and NRT6.3) was upregulated in both KZ15 and SZ7 under N-starvation.

It has been reported that SiMYB3 takes part in the growth and development of lateral roots and elongation of main roots mediated by low nutritional stress in millet; it also plays an important regulatory role in the signaling pathways of nitrogen, phosphorus, and potassium (Hu et al., 2015). A total of $235 \mathrm{MYB}$ transcription factors were found in this study. Two MYB transcription factors were especially upregulated in KZ15 under different nitrogen treatments, indicating that MYB transcription factors may play an important role in the process of low-nitrogen stress response in sorghum.

\section{Conclusion}

To analyze the molecular mechanism of sorghum response to low nitrogen, the phenotypic and transcript profiling of different resistant varieties were studied. The results showed that the content of chlorophyll and the activity of NR and GS of SZ7 
were more sensitive than that of KZ15 under low-N stress. A total of 1809 and 2657 DEGs were identified from KZ15 and SZ7.

We suggest that MYB transcription factors may play an important role in regulating the expression of genes related to nitrogen stress response. The ERF, bHLH, WRKY, and NF-Y families of transcription factors could also be involved in the response to low nitrogen tolerance in sorghum. In the tolerant cultivar KZ15, 389 upregulated genes were found functionally involved in low-N tolerance. These 389 genes are mainly involved in nitrogen metabolism, defense response, oxidative stress response, and flavonoid metabolism. We speculate that three nitrate transporter genes (NRT2.4, NRT3.1, and NRT4.5) and ten transcription factors may play an important role in improving the nitrogen use efficiency of sorghum. Overexpression of these genes may improve the tolerance of sensitive sorghum genotypes to nitrogen starvation. The expression patterns and regulation mechanisms of these genes associated with the tolerance to low nitrogen will be analyzed in future. These genes can also be used as candidate genes for improving sorghum tolerance to low nitrogen by genetic engineering, and lay a foundation for the breeding of new sorghum varieties with low nitrogen tolerance.

\section{REFERENCES}

[1] Anders, S., Huber, W. (2010): Differential expression analysis for sequence count data. Genome biology 11(10): R106.

[2] Ding, Y., Luo, W., Xu, G. (2006): Characterisation of magnesium nutrition and interaction of magnesium and potassium in rice. - Annals of Applied Biology 149(2): 111-123.

[3] Gallais, A., Hirel, B. (2004): An approach to the genetics of nitrogen use efficiency in maize. - Journal of Experimental Botany 55: 295-306.

[4] Gelli, M., Konda, A. R., Liu, K., Zhang, C., Clemente, T. E., Holding, D. R., Dweikat, I. M. (2017): Validation of QTL mapping and transcriptome profiling for identification of candidate genes associated with nitrogen stress tolerance in sorghum. - BMC Plant Biol. 17: 123 .

[5] Han, S. F., Li, S. W., Wu, L. Q., Wen, H. D., Xiao, K. (2007): Responses and corresponding physiological mechanisms of different wheat varieties in their nitrogen efficiency and nitrogen uptake to nitrogen supply. - Chinese Journal of Applied Ecology 18(4): 807-812.

[6] Himanen, K., Vuylsteke, M., Vanneste, S., Vercruysse, S., Boucheron, E., Alard, P., Chriqui, D., Montagu, M. V., Inze, D., Beeckman, T. (2004): Transcript profiling of early lateral root initiation. - Proc Natl Acad Sci USA 101: 5146-5151.

[7] Hu, L. (2015): Transcriptome analysis of Foxtail millet (Setaria italic) under low nitrogen stress and characteristics and functional identification of SiMYB3. - Master dissertation of Chinese Academy of Agricultural Sciences (in Chinese with English Abstract).

[8] Kim, D., Langmead, B. (2015): HISAT: a fast spliced aligner with low memory requirements. - Nat. Methods 12: 357-360.

[9] Kraiser, T., Gras, D. E., Gutierrez, A. G., Gonzalez, B., Gutierrez, R. A. (2011): A holistic view of nitrogen acquisition in plants. - J Exp Bot 62: 1455-66.

[10] Loudet, O., Chaillou, S., Merigour, P., Talbotec, J., Daniel, V. F. (2003a): Quantitative Trait Loci Analysis of nitrogen use efficiency in Arabidopsis. - Plant Physilology 131: 345-358. 
[11] Loudet, O., Chaillou, S., Krapp, A., Daniel, V. F. (2003b): Quantitative Trait Loci analysis of water and anion contents in interaction with nitrogen availability in Arabidopsis thaliana. - Genetics 163: 711-722.

[12] Ma, J. (2014): The morphological and physiological characteristics of sorghum under low phosphorus and nitrogen, and the study of sorghum microRNA under low nitrogen stress. - Ph.D. dissertation of Shanxi Agricultural University (in Chinese with English Abstract).

[13] Obara, M., Sato, T., Sasaki, S., Kashiba, K., Nagano, A., Nakamura, I., Ebitani, T., Yano, M., Yamaya, T. (2004): Identification and characterization of a QTL on chromosome 2 for cytosolic glutamine synthetase content and panicle number in rice. - Theor Appl Genet 110: 1-11.

[14] Presterl, T., Groh, S., Landbeck, M., Seitz, G., Schmidt, W., Geiger, H. H. (2010): Nitrogen uptake and utilization efficiency of European maize hybrids developed under conditions of low and high nitrogen input. - Plant Breeding 121(6): 480-486.

[15] Rajasekhar, V. K., Mohr, H. (1986): Appearance of nitrite reductase incotyledons of the mustard (Sinapis alba L.) seedling as affected by nitrate, phytochrome and photooxidative damage of plastids. - Planta 168(3): 369-376.

[16] Rosa, R. M., Rosa, Y., Rosa, J. J., Rosa Jr, E. J., Silva, E. F., Martinez, M. A., Rosa, D. B. C. J., Soares, J. S. (2014): Influence of nitrogen, potassium and phosphate fertilizers on quality and longevity of gladiolus. - Revista Brasileira de Horticultura Ornamental 20(2): 143-153.

[17] Wang, X. K. (2006): Principles and Techniques of Plant Physiology and Biochemistry Experiments. - Higher Education Press, Beijing.

[18] Xiong, S. P., Wu, K. Y., Wang, X. C., Zhang, J., Du, P., Wu, X., Ma, X. M. (2016): Analysis of root absorption characteristics and nitrogen utilization of wheat genotypes with different N efficiency. - Scientia Agricultura Sinica 49(12): 2267-2279.

[19] Zeng, J. M., Cui, K. H., Huang, J. L., He, F., Peng, S. B. (2007): Responses of physiobiochemical properties to $\mathrm{N}$-fertilizer application and its relationship with nitrogen use efficiency in rice (Oryza sativa L.). - Acta Agronomica Sinica 33(7): 1168-1176. 